PROCEEDINGS OF THE

AMERICAN MATHEMATICAL SOCIETY

Volume 28, Number 2, May 1971

\title{
KLEIN BOTTLES IN CIRCLE BUNDLES
}

\author{
JOHN W. WOOD
}

ABstract. We prove that the Klein bottle embeds in the total space $E$ of an orientable $S^{1}$-bundle over an orientable 2-manifold $M$ if and only if $M=S^{2}$ and $E=S^{1} \times S^{2}$ or the lens space $L(4,1)$.

In this note we apply results of [1] to generalize a result given there concerning the embedding of the Klein bottle.

Proposition. The Klein bottle embeds in the total space $E$ of an orientable $S^{1}$-bundle over an orientable 2-manifold $M$ if and only if $M=S^{2}$ and $E=S^{1} \times S^{2}$ or the lens space $L(4,1)$.

To show $M=S^{2}$ we use the following result of [1]:

THEOREM [1, \$4.1]. Let $i: K \rightarrow E$ be an embedding of a nonorientable $(n-1)$-manifold $K$ in an orientable n-manifold $E$. Suppose that $\alpha \in \pi_{1}(K)$ reverses orientation. Then for $\beta \in \pi_{1}(E), \beta^{-1} i_{*}(\alpha) \beta \in i_{*}\left(\pi_{1}(K)\right)$ implies $\beta \in i_{*}\left(\pi_{1}(K)\right)$.

Assume $M \neq S^{2}$, so $\pi_{2}(M)=0$. In the exact sequence of the fibration

$$
\cdots \rightarrow 0 \rightarrow \pi_{1}\left(S^{1}\right) \rightarrow \pi_{1}(E) \stackrel{p_{\#}}{\rightarrow} \pi_{1}(M) \rightarrow 0
$$

the generator of $\pi_{1}\left(S^{1}\right)$ is mapped to an element $g$ in the center of $\pi_{1}(E)$. (Since $E$ is trivial over the 1-skeleton of $M$, the inverse image of any circle in $M$ is a torus in $E$. Hence $g$ commutes with a basis for $\pi_{1}(E)$.) By the theorem $g$ is in the image of $i_{f}$. Let $\pi_{1}(K)$ $=\left\{\alpha, \beta: \alpha \beta \alpha^{-1}=\beta^{-1}\right\} ; \alpha$ is the orientation reversing element. Then $g=i_{\ddagger}\left(\alpha^{j} \beta^{k}\right)$ for some integers $j, k$. Since $\alpha \beta^{k} \alpha^{-1}=\beta^{-k}$, we have

$$
g i_{f}\left(\alpha^{-j+1}\right) g i_{\sharp}\left(\alpha^{-j-1}\right)=i_{\sharp}\left(\alpha^{j} \beta^{k} \alpha^{-j+1} \alpha^{j} \beta^{k} \alpha^{-j-1}\right)=1 .
$$

Therefore $g^{2}=i_{*}\left(\alpha^{2 j}\right) . p t(g)=0$ and $\pi_{1}(M)$ is torsion free, so $p_{t+i}(\alpha)$ $=0$. Therefore $i_{f}(\alpha)=g^{m}$ and is in the center of $\pi_{1}(E)$. But then by the theorem $i_{f}$ is onto. Thus $p \neq i_{*}(\beta)$ generates $\pi_{1}(M)$ which contradicts $M \neq S^{2}$.

To complete the proof of the proposition recall that the total space $E$ of an orientable $S^{1}$-bundle over $S^{2}$ is the lens space $L(k, 1)$ or $S^{1} \times S^{2}$ (the case $k=0$ ). By $[1, \S 6]$ a nonorientable surface of genus $g$ embeds

Received by the editors June $11,1970$.

AMS 1969 subject classifications. Primary 5720, 5570.

Key words and phrases. Embedding, Klein bottle, $S^{1}$-bundle over 2-manifold. lens space.

Copyright (c) 1971, ANerican Mathematical Society 
in $L(k, 1)$ if and only if $k$ is even, $g \equiv k / 2(\bmod 2)$, and $g \geqq k / 2$. Thus the Klein bottle, which has genus 2 , embeds only in $L(4,1)$ and $S^{1}$ $\times S^{2}$.

If $S^{1} \times S^{2}$ is pictured as a family of 2 -spheres parameterized by $\theta, 0 \leqq \theta<2 \pi$, then the surface swept out by a meridian rotated about the poles by $\theta / 2$ is a Klein bottle.

In the $x, y$-plane let $S$ be the square with vertices at $( \pm 1,0)$ and $(0, \pm 1) . L(4,1)$ is obtained from the suspension from $(0,0, \pm 1)$ of $S$ in $R^{3}$ by identifying certain points of the boundary, cf. [2, p. 223]. The surface $z=x y$ gives an embedding of the Klein bottle.

\section{REFERENCES}

1. G. E. Bredon and J. W. Wood, Non-orientable surfaces in orientable 3-manifolds, Invent. Math. 7 (1969), 83-110. MR 39 \#7616.

2. P. J. Hilton and S. Wylie, Homology theory: An introduction to algebraic topology, Cambridge Univ. Press, New York, 1960. MR 22 \#5963.

Institute for Advanced Study, Princeton, New Jersey 08540 